\title{
Culprit-Only Artery Versus Multivessel Disease
}

\author{
Valeria Paradies and Pieter C. Smits
}

\subsection{Introduction}

Primary percutaneous coronary intervention (pPCI) is the treatment of choice in patients presenting with ST-segment elevation myocardial infarction (STEMI). In contemporary practice, among patients who present to the hospital with STEMI, between 40 and $65 \%$ have concurrent multi-vessel (MV) coronary artery disease (CAD), a combination of a thrombotic culprit lesion and one or more significant (50\% or more diameter stenosis) non-culprit lesions in other coronary artery territories on coronary angiography. Optimal management of these non-culprit lesions in this setting is still a matter of debate. STEMI patients with MV CAD are at higher risk of recurrent cardiovascular events. However, PCI of bystander lesions during pPCI can bring potential complications. The presence of MV CAD in STEMI patients often poses therapeutic dilemma for interventional cardiologists as there are multiple possible strategies and controversial data. Besides clinical relevance, as the burden of cardiovascular disease affects hospital systems around the world, there is growing interest to examine and improve the various treatment strategies involved in the management of STEMI with MV CAD.

\subsubsection{Revascularization Strategies for STEMI Patients with MV CAD}

Revascularization strategies for non-culprit lesions in haemodynamically stable patients after pPCI currently vary from an aggressive approach with the PCI of all

\author{
V. Paradies \\ National Heart Research Institute Singapore, National Heart Centre, Singapore, Singapore \\ P. C. Smits $(\square)$ \\ Department of Interventional Cardiology, Maasstad Hospital, Rotterdam, Netherlands \\ e-mail: smitsp@maasstadziekenhuis.nl


Table 13.1 Factors influencing management of STEMI patients with MV CAD undergoing pPCI

\begin{tabular}{l|l|l}
\hline Clinical factors & Procedural factors & Lesion characteristics \\
\hline Age & Complexity & Complexity/SYNTAX score \\
Comorbidities & Duration/contrast & Number of vessel disease \\
Haemodynamic stability & Final result & LM involvement \\
LV function & & CTO calcifications \\
Renal function & & \\
Diabetes & & \\
\hline
\end{tabular}

significant non-culprit lesions during the index intervention to a very conservative approach with culprit lesion-only treatment and only symptom-driven or ischaemiadriven non-culprit PCI during index hospitalization or after discharge (Table 13.1).

In general three PCI strategies can be identified:

1. Culprit vessel-only pPCI with optimal medical therapy for bystander lesions and PCI of the non-culprit arteries only for spontaneous angina or myocardial ischaemia on stress testing

2. MV PCI at the time of pPCI, guided by angiography or FFR

3. Culprit vessel-only pPCI, followed by angiography or FFR-driven staged PCI of non-culprit arteries during the index hospitalization or after hospital discharge

Incomplete revascularization of STEMI patients presenting with MV CAD has been associated with worse early and late prognosis. The culprit vessel-only PCI strategy may reduce the contrast volume and risk of PCI complications but has been associated with increased risk of repeat revascularization and potential reduction in left ventricle ejection fraction (LVEF) recovery. There are several potential advantages of performing single-stage MV PCI. Firstly, the complete restoration of myocardial blood supply in the acute phase may increase myocardial salvage in hibernating myocardium, therefore improving LVEF. Secondly, this strategy decreases the risk of access site vascular complications associated with repeat vascular punctures. Thirdly, single-stage MV PCI reduces hospitalization with a relevant impact on the healthcare costs. Finally, complete revascularization has been associated with an improved prognosis after PCI: achieving complete revascularization decreases the risk of a future acute coronary syndrome (ACS) or revascularization procedures and improves prognosis. Naturally, there are safety concerns associated with this strategy including prolongation of the interventional procedure, contrast overload and increased radiation exposure. Moreover, the occurrence of procedural complications during non-culprit lesion PCI may further depress LV function which is already compromised from the initial STEMI event. Furthermore, the risk of abrupt vessel closure and stent thrombosis may be increased in this setting due to pro-thrombotic and pro-inflammatory state, while the risk of jeopardizing remote viable myocardium (distal embolization, no reflow, side branch occlusion, loss of collateral circulation) may result in haemodynamic instability.

Despite the additional risks related to PCI access site and additional costs, the staged PCI strategy allows more time to appropriately investigate and weigh on the risks and benefits of non-culprit lesion intervention. 


\subsubsection{Randomized Trials}

There are a number of key clinical trials which provide the wealth of guidance with regard to timing of non-culprit lesion intervention in the setting of STEMI.

\subsubsection{PRAMI}

In the Preventive Angioplasty in Acute Myocardial Infarction (PRAMI) trial, a total of 465 patients were randomly assigned to culprit-only revascularization $(n=231)$ and complete revascularization during the index procedure $(n=234)$. The study defined the presence of MV CAD as a non-infarcted-related artery lesion of $\geq 50 \%$ by angiography. Staged revascularization in the asymptomatic patients was discouraged. Patients in cardiogenic shock, unable to provide consent, with previous CABG or non-infarct stenosis of $\geq 50 \%$ in the left main or the ostia of both the left anterior descending and circumflex arteries, or if the only non-culprit stenosis was a chronic total occlusion (CTO), were excluded. After a mean follow-up of 23 months, this study reported a $65 \%$ reduction in the primary endpoint (cardiovascular death, new myocardial infarction (MI) and refractory angina defined as angina despite medical therapy supported by evidence of objective ischaemia) in complete revascularization group compared to the culprit-only revascularization group. The study was discontinued early by the data safety monitoring board, due to a significant difference in primary endpoint in favour of complete revascularization which was mainly driven a significant reduction in refractory angina and non-fatal MI; however, no significant reduction in mortality was shown.

Although the study reported convincingly positive study results in favour of single-stage MV PCI, the study had little impact on clinical practice. Several concerns have been raised: the randomization process was not stratified for timing of intervention in relation to symptom onset, nor for site of infarction; the study enrolment included less than $50 \%$ of screened patients with possible selection bias; no more information were provided on the non-culprit lesions (i.e. QCA, TIMI flow or lesion characteristics). Moreover, the infarct-related artery (IRA) only revascularization group in this study demonstrated a higher proportion of diabetic patients and anterior MI, which might have influenced the worse prognosis in this group. Finally, this study compared only the most aggressive to the most conservative strategy.

\subsubsection{CvLPRIT}

The Complete versus Lesion-only Primary PCI (CvLPRIT) trial randomized 296 STEMI patients presenting with MV CAD to culprit-only strategy $(n=146)$ or complete revascularization $(n=150)$. Patients were excluded in case of age $<18$ years, clear contraindication to MV pPCI according to operator judgement, prior MI or CABG, cardiogenic shock, ventricular septal rupture or moderate/severe mitral regurgitation, chronic kidney disease, thrombosis of a previously stented artery and chronic total occlusion of the only non-culprit.

This study reported a 55\% reduction of the primary composite endpoint of allcause mortality, recurrent MI, heart failure and ischaemia-driven revascularization 
by PCI or CABG within 12 months in the complete revascularization group as compared to the culprit-only group. The investigators found also lower incidence of individual components of the primary endpoint in the complete revascularization group, though not statistically significant. Complete revascularization was performed either at the time of index procedure (64\%) or before hospital discharge (36\%). The former group showed a trend of greater benefit in terms of MACE incidence as compared to the latter. There was no increase in stroke, major bleeding or contrast-induced nephropathy with complete versus culprit-only revascularization. A pooled analysis of PRAMI and CULPRIT showed a significant reduction in individual endpoints of $\mathrm{CV}$ death, recurrent $\mathrm{MI}$ and repeat revascularization associated with complete revascularization approach.

\subsubsection{DANAMI-3 PRIMULTI}

In The Third Danish Study of Optimal Acute Treatment of Patients with STEMI: Primary PCI in Multivessel Disease (DANAMI-3 PRIMULTI), 627 patients were randomized to receive culprit lesion-only treatment $(n=314)$ versus fractional flow reserved (FFR)-guided complete revascularization $(n=312)$. Patients with an angiographic diameter stenosis $>50 \%$ in one or more non-IRA were enrolled and randomized after successful PCI of the culprit lesion. Patients intolerant to contrast media, relevant anticoagulant or antithrombotic drugs, in cardiogenic shock, with stent thrombosis, increased bleeding risk or indication for CABG were excluded from the trial. The FFR-guided staged revascularization was performed 2 days after the index procedure and during the index hospitalization.

One-third of patients within the complete revascularization group had FFR values $>0.80$ and did not receive PCI. The investigators demonstrated a significant $44 \%$ reduction in the composite primary endpoint of death, MI or ischaemia-driven revascularization within 12 months in the FFR-guided complete revascularization group, which was largely driven by a $69 \%$ reduction of ischaemia-driven revascularization of the non-IRAs. There were no significant differences in the all-cause mortality or non-fatal MI rates between the two groups. This trial was not powered to evaluate an impact on hard outcomes (such as death and MI).

\subsubsection{COMPARE-ACUTE Trial}

The Comparison Between FFR-Guided Revascularization Versus Conventional Strategy in Acute STEMI Patients with MVD ${ }^{\wedge}($ COMPARE-ACUTE) trial enrolled 885 STEMI patients with MV CAD. After successful revascularization of the culprit artery, patients were randomly assigned in a ratio of 1:2 to FFR-guided complete revascularization $(n=295)$ and culprit-only revascularization $(n=590)$. The most important exclusion criteria were left main CAD, chronic total occlusion, severe stenosis with a Thrombolysis in Myocardial Infarction (TIMI) flow grade of 2 or less in the non-IRA, a suboptimal result or complication after treatment of IRA, severe valve dysfunction and Killip class III or IV. Complete revascularization was performed in lesions with FFR $\leq 0.80$, preferably during the index procedure, but could be delayed within $72 \mathrm{~h}$ (complete PCI was performed during index procedure in $83.4 \%$ patients). Primary endpoint of the study was the composite of all-cause 
mortality, non-fatal MI, any revascularization and cerebrovascular events (MACCE) at 12 months. FFR-guided revascularization treatment was associated with lower rate of MACCE compared to culprit-only revascularization, which seems to be driven mainly by the need for repeat revascularization. Similar to previous studies, COMPARE-ACUTE trial was not powered to detect differences in low-frequency events, such as death, re-infarction and stroke. However, in contrast with DANAMI3PRIMULTI, this study investigated the role of FFR-guided revascularizations during the index procedure, supporting this strategy as safe and cost-saving, from both a patient and economic perspective.

One main criticism of the trial was potential operator bias concerning the decision process about staging the revascularization of non-IRAs beyond the 45 days. In the IRA-only group, urgent revascularizations performed within this time window but after the index procedure or further revascularizations performed thereafter were indeed counted as events. Moreover, in the same treatment group, the indication to treat the non-culprit lesions was heterogeneous and based on detection of ischaemia, symptoms or clinical judgement. However, the rate of MACCE at 1 year remained significantly lower with the complete revascularization strategy even extending the treatment window from 45 to 60 or 90 days and excluding nonurgent revascularizations.

\subsubsection{Safety of Complete Revascularization}

The recommendation against PCI of non-culprit lesions was largely driven by results of non-randomized observational studies with conflicting results. These recommendations arose from historical safety concerns that included an increased potential risk for procedural complications. However, more complete acute revascularization in patients with STEMI may be safer in the current era due to advances in stent technology and antiplatelet therapy. Recent evidences suggest that potential longer procedural time or increased use of contrast in complete revascularization does not translate into an increased risk of adverse events. A pooled analysis of PRAMI, CULPRIT and the trial by Politi et al. found no increase in cerebrovascular (CVA) events, bleeding or contrast-induced nephropathy associated with PCI of non-culprit lesions performed at the time of index procedure. DANAMI3PRIMULTI study demonstrated similar rates of peri-procedural MI, stroke, contrastinduced nephropathy or bleeding between IRA-only and complete revascularization treatment. In a similar way, the recent COMPARE-ACUTE found no differences in terms of peri-procedural MI and bleeding between the two strategies.

\subsubsection{Timing of Revascularization}

The optimal timing for revascularization of the non-culprit lesions remains extremely debated. Potential options include performing complete revascularization during index procedure or as planned elective procedure during index 
hospitalization or after discharge. To date there are no RCTs investigating both staged and immediate complete revascularization and analysing them separately. PRAMI specifically compared single-stage MV PCI to culprit-only strategy, while DANAMI3- PRIMULTI analysed only staged versus culprit-only revascularization. The two RCTs CvLPRIT and COMPARE-ACUTE were designed to perform complete revascularization in the acute phase, but allowed staged revascularization, which happened in a minority of the cases. Although all RCT trials show an advantage of immediate or early staged complete revascularization guided by angiography or FFR in comparison to culprit-only treatment, still uncertainties exist about when the complete revascularization should be performed. Looking at the time to event curves in all the above-mentioned trials, we can observe that the event curves start to diverge from the outset, presumably indicating that an immediate or very early revascularization strategy is likely to be beneficial. This assumption is complemented by a meta-analysis by Wang et al. comparing complete revascularization during the index procedure with staged revascularization that found a significantly lower incidence of MACE, all-cause death and/or MI, non-fatal MI and repeat revascularization associated with the former strategy.

Interestingly, the benefit of the immediate complete revascularization strategy has not been confirmed in other meta-analyses and in the various published observational studies. In the post hoc analysis of HORIZONS-AMI trial, MV PCI during index procedure was associated with an increased all-cause and cardiovascular mortality compared to the staged MV PCI in a total of 668 STEMI patients. Similarly, a recent meta-analysis by Tarantini et al. demonstrated that a staged MV revascularization strategy may improve both early and late survival. Moreover, a propensitymatched analysis of 3984 patients presenting with STEMI and MV disease suggested an improved survival and improved MACE with culprit-only PCI during the index procedure. The confusion is compounded by a paired and network meta-analysis of 14 studies including 40,280 patients and compared three timing strategies in STEMI patients with MV disease: staged PCI (defined as separate procedure during index admission or within 1 month of the primary PCI), immediate complete revascularization during index pPCI procedure and culprit-only PCI. This analysis found lowest short- and long-term mortality rates in patients undergoing staged complete revascularization.

Overall, the above studies suggest staged revascularization as the best option. Nevertheless, these data are from prior observational studies and meta-analyses of revascularization strategies derived from an extremely heterogeneous set of inclusion criteria, study protocols, PCI techniques, timing of MV PCI and comparator groups. Also with divergent analytical methods and variable endpoints, definite conclusions are difficult to draw.

\subsubsection{Trials on the Horizon}

The large ongoing randomized, Complete versus Culprit-only Revascularization to Treat Multivessel Disease After Primary PCI for STEMI (COMPLETE) trial will 
enrol a total of 3900 STEMI patients with MV disease and is estimated to be completed in December 2018. Patients are randomized to receive either staged revascularization or culprit-only revascularization, on top of optimal medical therapy (including low-dose aspirin and ticagrelor in both arms and FFR guidance in intermediate lesions (50-70\% diameter stenosis)) in the complete revascularization arm. The FULL REVASC trial from Sweden is another large-scale trial randomizing 4052 MV-STEMI or high-risk MV-NSTEMI patients between FFR-guided complete revascularization in the acute or staged phase and angiography-guided. The primary endpoint of both trials are the combination of recurrent myocardial infarction and all death (FULL REVASC) or cardiovascular death (COMPLETE) and will provide final answers with hard endpoints as to whether staged revascularization is better than culprit-only revascularization or FFR guidance is better compared to angiography in acute MI patients with MV disease.

\subsection{State of the Art: Current Recommendations}

Both the 2014 European Society of Cardiology guidelines and 2013 American College of Cardiology/American Heart Association guidelines did not recommend revascularization of non-culprit lesions in the setting of STEMI unless complicated by cardiogenic shock. These recommendations were influenced strongly by safety concerns based on observational studies. The publication of larger scale RCTs has prompted ACC/AHA to change the recommendation for complete revascularization to class $\mathrm{IIb}$ in the recent 2015 ACC/AHA/SCAI Focused Update on Primary Percutaneous Intervention for Patients with ST-Elevation Myocardial Infarction. These guidelines specifically allow consideration of single-stage MV PCI in selected patients, either at the time of pPCI or as a delayed, staged procedure. Similarly, 2017 ESC guidelines for the management of acute myocardial infarction in patients presenting with STEMI recommend that revascularization of non-IRA should be considered in STEMI patients with MV CAD at index or before hospital discharge (class IIa). The optimal timing of revascularization (immediate vs. staged) has not been adequately investigated; therefore, no recommendation is provided in these guidelines.

\subsection{Role of Fractional Flow Reserve}

Most of the observational and randomized studies have so far used angiographic diameter stenosis severity to determine non-culprit lesions requiring PCI. Fractional flow reserve (FFR) has historically not been utilized in ACS due to concern that acute microvascular dysfunction might influence these results. It has been suggested that non-IRA stenosis severity may be acutely exaggerated as the result of circulating catecholamine-mediated vasoconstriction. Moreover, virtual histology intravascular ultrasound (VH-IVUS) analysis of non-culprit lesions in ACS patients has shown a greater prevalence of vulnerable plaques, with greater necrotic core and thin-cap fibroatheroma as compared with stable coronary lesions. 
Nevertheless, reliability of FFR of non-culprit lesions in the acute phase of STEMI has been extensively demonstrated. Ntalianis et al. reported the reproducibility of FFR measurements in 75 STEMI patients undergoing PCI when repeated at a mean of $35 \pm 4$ days post initial procedure. The same was observed by a similar study performed by Musto et al. when similar FFR and instantaneous wave-free ratio (iFR) values were obtained in the acute phase and 7 days later in $60 \mathrm{MV}$-STEMI patients undergoing pPCI. Moreover, the COMPARE-ACUTE trial demonstrated a discrepancy between angiographic and haemodynamically significant lesions in STEMI patients; approximately half of non-culprit lesions that were considered to be critical on coronary angiography were found to have an FFR value $>0.80$ and therefore not physiologically relevant.

Both DANAMI3-PRIMULTI and COMPARE-ACUTE trial showed that FFRguided PCI decreased acute and repeat revascularization rates, though did not impact mortality or re-infarction rates. However, performing FFR during index procedure, as investigated in COMPARE-ACUTE trial, was found to be safe and to reduce cost and risk associated with a delayed procedure, justifies a selective anatomically incomplete revascularization, expedites post-STEMI care and discharge, facilitates decision-making in the heart team and may offer reassurance to the patient.

\subsection{Prognostic Value of Complete Revascularization}

Although recent RCTs showed a benefit of composite MACE endpoints associated with complete revascularization as compared to culprit-only strategy, this was mainly driven by a reduction in ischaemia-driven revascularization. Of note, none of these trials was powered to evaluate prognostic clinical endpoints such as death and myocardial infarction. Interestingly, a meta-analysis of four RCTs including CvLPRIT, PRAMI, Politi and HELP-AMI for a total of 1044 patients that compared complete revascularization and culprit-only demonstrated significant reduction in long-term ( $\geq 1$ year) all-cause mortality, cardiovascular death and MI associated with the former treatment. A low degree of heterogeneity between the studies was reported for this meta-analysis. Despite all the limits related to differences of individual studies, these findings suggest a trend towards improved death and MI with complete revascularization. Further clarification is expected from the COMPLETE trial which will investigate the benefit of complete revascularization in terms of composite of cardiovascular death and MI as primary endpoint.

\subsection{STEMI with Multivessel Coronary Artery Disease Complicated by Cardiogenic Shock}

Cardiogenic shock is present in 6-12\% of cases with acute myocardial infarction, varying according to the population analysed and definition of cardiogenic shock. The presence of MV CAD has been reported in up to 60-70\% of STEMI patients complicated by cardiogenic shock. In the Should We Emergently Revascularize 
Occluded Coronaries for Cardiogenic Shock (SHOCK) trial, three-vessel CAD was found in $60 \%$ of patients undergoing PCI. MV CAD in STEMI patients complicated by cardiogenic shock has been found to be an independent predictor of in-hospital mortality.

Most of the current available data regarding the management of MV CAD in the context of cardiogenic shock are based on retrospective analysis of registries, therefore producing heterogeneous and conflicting results.

In the milestone SHOCK trial, 352 patients with acute myocardial infarction complicated by cardiogenic shock were randomized to receive either emergency revascularization with either PCI or CABG $(n=152)$ or initial medical stabilization $(n=150)$. Despite the high rate of intra-aortic balloon pump used in both groups, the investigators found no differences in terms of 30-day mortality and lower 6-month mortality rates in the complete revascularization group as compared to culprit-only treatment. This study showed that a strategy of early revascularization resulted in 6-year higher survival rates compared with initial medical stabilization. Retrospective sub-analysis of KAMIR registry evaluating 510 STEMI patients with cardiogenic shock and evidence of MV CAD at angiography revealed reduction of early mortality with complete revascularization. Similarly, a prospective observational study of 266 STEMI patients with cardiogenic shock showed improved 6-month survival associated with single-stage MV PCI strategy.

Results of the CULPRIT-SHOCK (Culprit Lesion-Only PCI Versus MV PCI in Cardiogenic Shock) trial have been recently published. This multicentre, open-label trial randomized 706 patients with cardiogenic shock and evidence of MV CAD at index angiography to receive either culprit vessel revascularization (during index procedure with possible staged revascularization) or single-stage MV PCI. The use of intra-aortic balloon pump and mechanical support device use was left at operator's discretion. The 30-day risk of a composite of death or severe renal failure leading to renal replacement therapy was lower among those who initially underwent PCI of the culprit lesion-only than among those who underwent immediate MV PCI. One potential limitation of this study is advocating attempts at CTO revascularization in the acute setting, which is usually performed only with evidence of ischaemia and viability demonstrated in the CTO territory.

Current ESC guidelines recommend that non-IRA PCI during the index procedure should be considered in STEMI patients with cardiogenic shock (Class IIA, Level of evidence $\mathrm{C}$ ). The ACC/AHA/SCAI guidelines recommend emergency revascularization with either PCI or CABG irrespective of the time delay from myocardial infarction onset in STEMI patients complicated by cardiogenic shock (Class I, Level of evidence B). The rationale behind complete revascularization is to improve perfusion to non-IRA territories in order to reverse the spiral of decline that characterizes this status. In the context of myocardial ischaemia, a pan-myocardial inflammatory process as well as systemic hypotension might impact on the entire coronary circulation and exacerbate ischaemia in non-IRA lesions, leading to further coronary hypoperfusion and impaired myocardial function. However, despite current recommendations, multiple registries have shown that only one-fourth to one-third of STEMI patients with cardiogenic shock and evidence of MV CAD undergo immediate MV PCI. 


\subsection{Clinical Practice}

The recently reported RCTs have shown potential benefit of complete revascularization strategy which have led to guideline updates. These studies reflect more contemporary clinical practice where the use of DES, radial access and more effective P2Y12 inhibitors has improved clinical outcomes and reduced procedure-related complications. These findings have shed new light on the potential management of such patients. The use of FFR in this context has been proposed as a safe tool able to guide a functionally complete revascularization.

However, there are still unsolved issues with regard to the optimal timing of intervention as the current data present conflicting evidence. Moreover, the recent RCTs have shown improved MACE outcomes with complete revascularization mostly driven by need for repeat revascularization but are underpowered to determine hard clinical endpoints of death and MI. Furthermore, whether the goal of complete revascularization should be the treatment of ischaemia-related lesions or vulnerable plaques prone to thrombosis has yet to be determined.

Despite the available evidence and ongoing trials, defining a common strategy for all STEMI patients with MVD remains challenging. These patients are extremely heterogeneous, and any revascularization strategy should be individualized based on patient and lesion characteristics. Physiological evaluation of non-culprit lesions should be encouraged in order to define appropriate revascularization strategy.

\subsection{Case Report}

\section{Valeria Paradies and Peter C. Smits}

A 58-year-old man, without cardiac history, was admitted to our hospital for acute onset of chest pain and diagnosis of inferior STEMI. The ECG showed clear ST elevation in inferior leads, and the coronary angiography confirmed occlusion of RCA (Fig. 13.1a). However, a clear image of thrombus was detected in the mid-LAD involving the ostium of a diagonal branch (Fig. 13.1b). Our strategy began with PCI of RCA with a drug-eluting stent (DES) $4.0 \times 12 \mathrm{~mm}$ (Fig. 13.2a). Due to plaque shift, a second $4.0 \times 18 \mathrm{~mm}$ DES was implanted proximal to the previous one (Fig. 13.2b). No reflow occurred but rapidly improved with i.c. verapamil injection. Despite the culprit lesion having been identified and successfully treated, a clear image of thrombus that was evident in the mid-LAD was concerning, although not immediately compromising flow or generating apparent rest ischaemia. As the culprit lesion was successfully treated in a relatively short time and the patient remained haemodynamically stable despite transient no reflow, we considered the option to proceed with immediate PCI of LAD and diagonal branch, both of which were non-culprit lesions (despite the presence of obvious but non-occlusive thrombus)

A provisional approach was taken, and predilatation of the LAD with a $2.5 \times 10 \mathrm{~mm}$ balloon followed by DES $3.5 \times 23 \mathrm{~mm}$ implantation in mid-LAD was performed (Fig. 13.3a). After LAD stenting, plaque shift occurred towards the ostium of the diagonal (Fig. 13.3b). At this point, our decision-making process considered the 

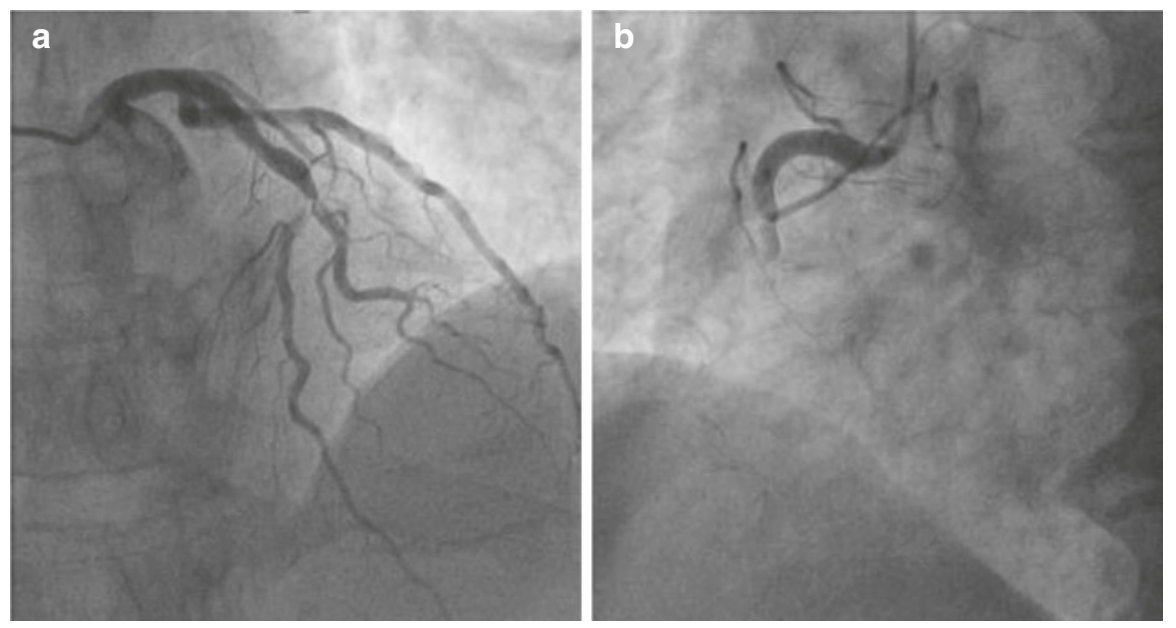

Fig. 13.1 (a) Left coronary angiography showed images of non-occlusive thrombus in the midLAD involving the origin of a relevant diagonal branch. (b) Right coronary angiography showed complete thrombotic occlusion of the right coronary artery in line with ECG findings of inferior STEMI
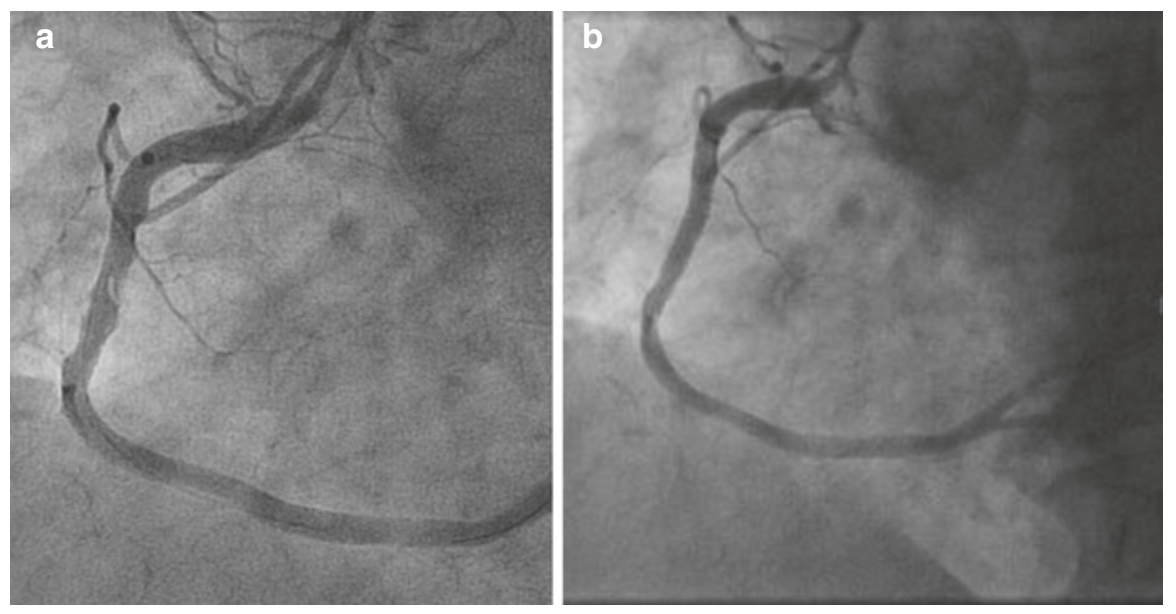

Fig. 13.2 (a) Proximal plaque shift after DES 4.0 $\times 12$ implantation on mid-RCA. (b) Final result after second stent implantation, i.c. verapamil administration and postdilatation of RCA lesion

potentially complicated issue of leading to a two-stent bifurcation PCI of a non-culprit vessel in a STEMI setting. However, given the degree of stenosis at ostium of the diagonal and calibre of this vessel, we decided to complete the procedure with a TAP stenting technique. A DES $2.75 \times 8 \mathrm{~mm}$ was implanted in the diagonal branch and final kissing balloon dilatation performed using oversized NC balloons (Fig. 13.3c). The patient remained haemodynamically stable and was discharged home a few days later. Although there is currently a vivid debate on complete revascularization during 

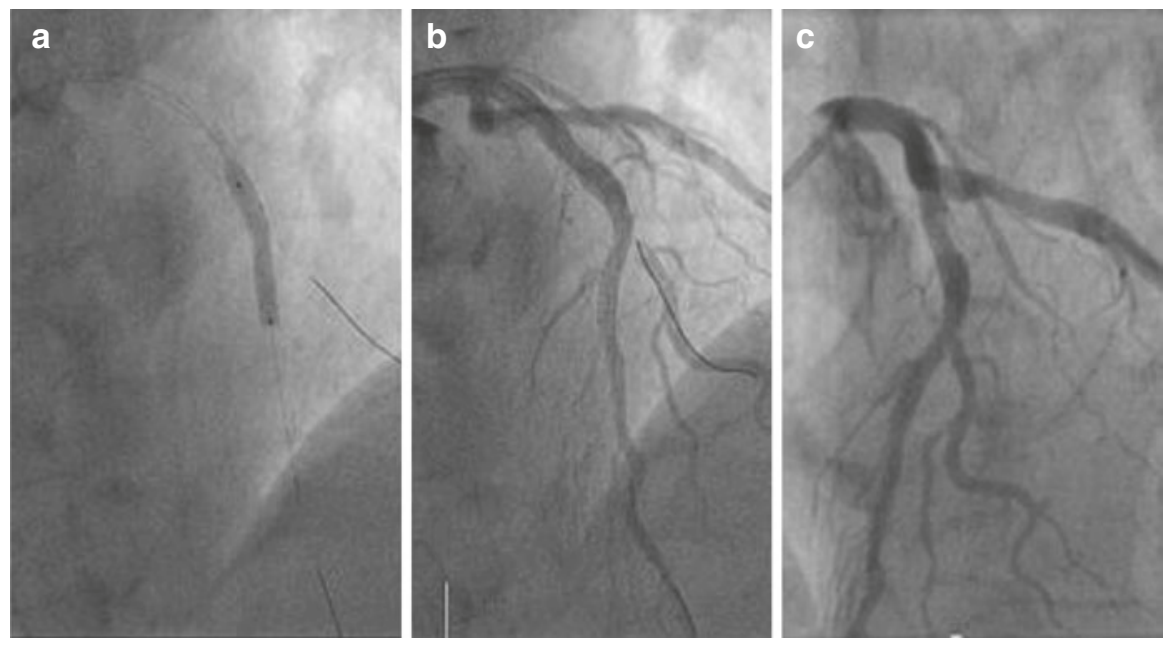

Fig. 13.3 (a) DES $3.5 \times 23 \mathrm{~mm}$ implantation on mid-LAD. (b) Result after LAD stenting. (c) Final result after D1 stenting with TAP technique and final kissing balloon

pPCI, this case highlights how the risk/safety balance of non-culprit PCI was steered by the angiographic findings of high thrombus burden in mid-LAD, which if left untreated may have had severe consequences.

\section{Further Readings}

Engstrøm T, Kelbæk H, Helqvist S, et al. Complete revascularisation versus treatment of the culprit lesion only in patients with ST-segment elevation myocardial infarction and multivessel disease (DANAMI-3 - PRIMULTI): an open-label, randomised controlled trial. Lancet. 2015;386:665-71.

Gershlick AH, Khan JN, Kelly DJ, et al. Randomized trial of complete versus lesion-only revascularization in patients undergoing primary percutaneous coronary intervention for STEMI and multivessel disease: the CvLPRIT trial. J Am Coll Cardiol. 2015;65:963-72.

Smits PC, Abdel-Wahab M, Neumann FJ, et al. Fractional flow reserve-guided multivessel angioplasty in myocardial infarction. N Engl J Med. 2017;376:1234-44.

Wald DS, Morris JK, Wald NJ, et al. Randomized trial of preventive angioplasty in myocardial infarction. N Engl J Med. 2013;369:1115-23.

Open Access This chapter is licensed under the terms of the Creative Commons Attribution 4.0 International License (http://creativecommons.org/licenses/by/4.0/), which permits use, sharing, adaptation, distribution and reproduction in any medium or format, as long as you give appropriate credit to the original author(s) and the source, provide a link to the Creative Commons license and indicate if changes were made.

The images or other third party material in this chapter are included in the chapter's Creative Commons license, unless indicated otherwise in a credit line to the material. If material is not included in the chapter's Creative Commons license and your intended use is not permitted by statutory regulation or exceeds the permitted use, you will need to obtain permission directly from the copyright holder. 